\title{
Plasma Apelin concentrations in Patients with Polyuria-Polydipsia Syndrome
}

Sandrine A. Urwyler ${ }^{*}$, Katharina Timper ${ }^{*}$, Wiebke Fenske ${ }^{3}$, Nadia de Mota ${ }^{4}$, Anne Blanchard ${ }^{5}$, Felix Kühn ${ }^{6}$, Nica Frech ${ }^{1}$, Birsen Arici ${ }^{7}$, Jonas

Rutishauser ${ }^{8}$, Peter Kopp ${ }^{9}$, Christoph Stettler ${ }^{6}$, Beat Müller ${ }^{10}$, Mira Katan ${ }^{11}$, Catherine Llorens-Cortes ${ }^{4 \dagger}$ and Mirjam Christ-Crain ${ }^{1 \dagger}$ "equally contributing first authors, 'equally contributing last authors

${ }^{1}$ Clinic of Endocrinology, Diabetes and Metabolism, Department of Clinical Research, University Hospital Basel, Switzerland; ${ }^{2}$ Max-Planck-Institute for Metabolism Research, Cologne, Germany; ${ }^{3}$ Integrated Research and Treatment Center for Adiposity Diseases, Leipzig University Medical Center, Germany; 4 Laboratory of Central Neuropeptides in the Regulation of Body Fluid Homeostasis and Cardiovascular Functions, CIRB, College de France, Paris, France ${ }^{5}$ Assistance publique des hôpitaux de Paris, European Georges Pompidou Hospital, Clinical Investigation Center Paris, France; ${ }^{6}$ Division of Endocrinology, Diabetes and Clinical Nutrition, University Hospital Bern - Inselspital, Switzerland ; ${ }^{7}$ Department of Internal Medicine, Spital Rheinfelden, Switzerland; ${ }^{8}$ University Clinic of Internal Medicine, Kantonsspital Baselland, Bruderholz, Switzerland; ${ }^{9}$ Division of Endocrinology, Metabolism and Molecular Medicine and Center for Genetic Medicine, Northwestern University, Chicago, USA; ${ }^{10}$ Division of Endocrinology, Diabetology and Metabolism, Medical University Clinic, Kantonsspital Aarau, Switzerland; ${ }^{11}$ Department of Neurology, University Hospital Zurich, Switzerland

\section{Background \& Aim}

\section{Background:}

Apelin and arginine-vasopressin (AVP) are antagonists in the regulation of body fluid and osmotic homeostasis and act oppositely in healthy volunteers.

In patients with SIADH apelin levels were inadequately low in comparison to high AVP levels.

$[1,2]$

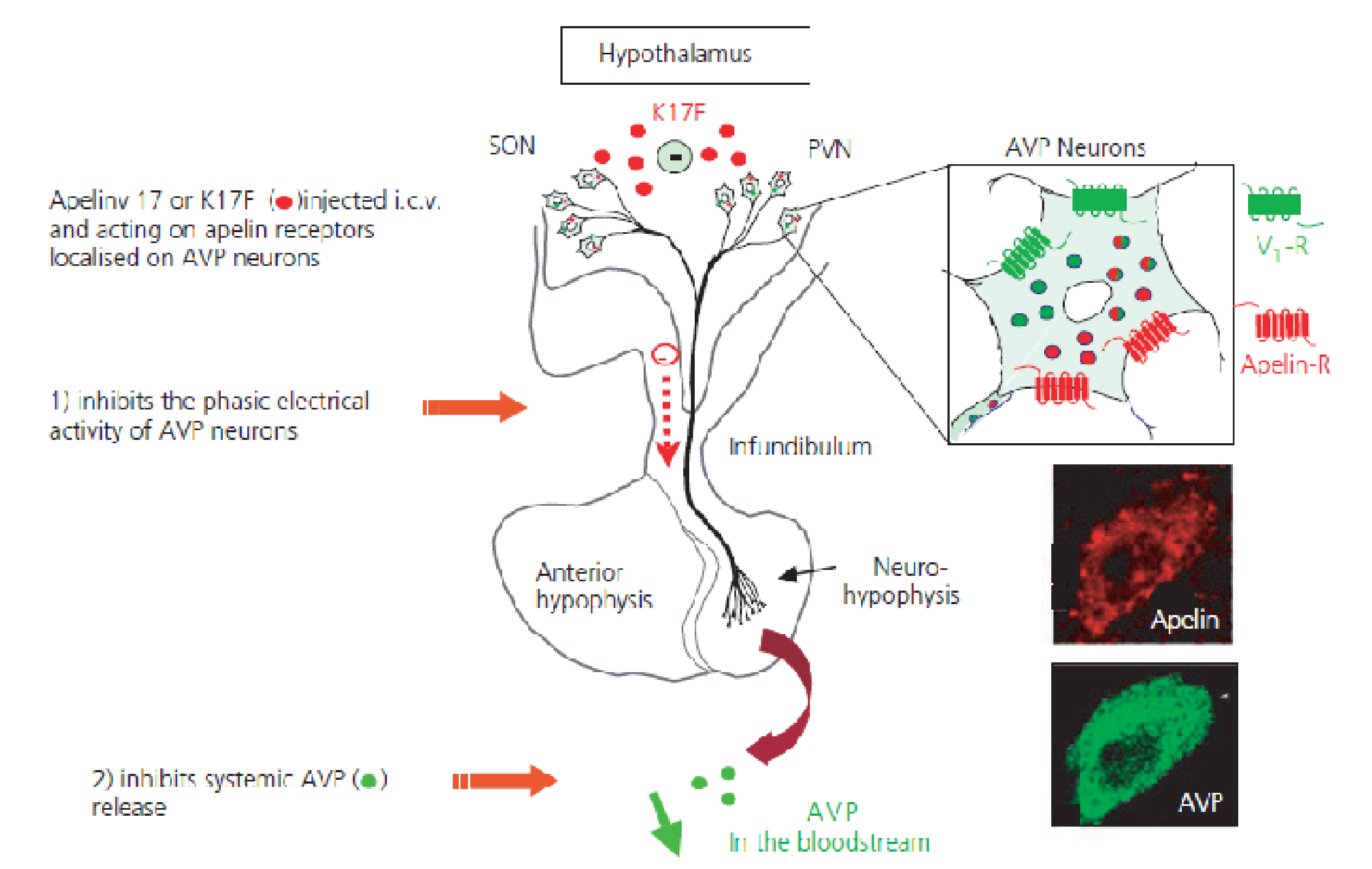

Aim:

To investigate plasma apelin levels and their relation to AVP/copeptin (surrogate marker for AVP) in patients with polyuria-polydipsia syndrome.

\section{Methods}

Plasma apelin and copeptin concentrations were measured in 41 patients with polyuria-polydipsia syndrome (15 central diabetes insipidus (cDI), 7 nephrogenic $\mathrm{DI}(\mathrm{nDI}), 19$ primary polydipsia (PP)) and were compared to those in 113 healthy volunteers.

\section{Results}

Table 1: Baseline Characteristics

\begin{tabular}{|c|c|c|c|c|c|}
\hline & $\begin{array}{c}\text { Healthy } \\
\text { volunteers } \\
(n=113)\end{array}$ & $\begin{array}{c}\text { Complete } \\
\text { central DI } \\
(n=15)\end{array}$ & $\begin{array}{l}\text { Primary } \\
\text { Polydipsia } \\
\text { (n=19) }\end{array}$ & $\begin{array}{c}\text { Complete } \\
\text { nephrogenic DI } \\
(n=7)\end{array}$ & p-value \\
\hline Age & $47(35 ; 61)$ & $50(42 ; 58)$ & $45(30 ; 50)$ & $61(55 ; 65)$ & 0.12 \\
\hline BMI $\left(\mathbf{k g} / \mathbf{m}^{2}\right)$ & $24(22 ; 26)$ & $25(23 ; 29)$ & $26(20 ; 29)$ & $26(25 ; 28)$ & 0.10 \\
\hline Sex, female & $58(51 \%)$ & $9(60 \%)$ & $13(68.4 \%)$ & $5(71.4 \%)$ & 0.43 \\
\hline History of a brain tumor & n.a. & $6(40 \%)$ & $1(5 \%)$ & $0(0 \%)$ & 0.02 \\
\hline History of trans-sphenoidal surgery & n.a. & $7(46.7 \%)$ & $1(5 \%)$ & $0(0 \%)$ & 0.02 \\
\hline Blood pressure systolic (mmHg) & $124(116 ; 134)$ & $121(115 ; 137)$ & $124(110 ; 131)$ & $137(117 ; 140)$ & 0.53 \\
\hline Heart rate (bpm) & $66(59 ; 74)$ & $70(67 ; 77)$ & $76(63 ; 81)$ & $83(79 ; 85)$ & 0.0005 \\
\hline $\begin{array}{l}\text { Clinical volume status: Hypo-/eu- } \\
\text { /hypervolaemic (euvolaemic \%) }\end{array}$ & n.a. & $\begin{array}{l}3 / 12 / 0 \\
(80 \%)\end{array}$ & $\begin{array}{l}2 / 14 / 0 \\
(87.5 \%)\end{array}$ & $\begin{array}{l}3 / 1 / 0 \\
(25 \%)\end{array}$ & 0.039 \\
\hline Hemoglobin (g/l) & n.a. & $140(130 ; 148)$ & $136(134 ; 143)$ & $128(115 ; 138)$ & 0.31 \\
\hline Hematocrit (I/l) & $0.41(0.39 ; 0.43)$ & $0.39(0.38 ; 0.44)$ & $0.40(0.38 ; 0.43)$ & $0.37(0.34 ; 0.4)$ & 0.13 \\
\hline Creatinine (umol/l) & $76(65 ; 84)$ & $75(63 ; 85)$ & $69(62 ; 74)$ & $146(125 ; 266)$ & $<0.0001$ \\
\hline Albumine (g/l) & n.a. & $39(34 ; 40)$ & $37(36 ; 42)$ & $37(32 ; 39)$ & 0.16 \\
\hline Plasma sodium (mmol/l) & $140(139 ; 141)$ & $142(142 ; 144)$ & $141(140 ; 142)$ & $143(142 ; 146)$ & $<0.001$ \\
\hline Plasma osmolality (mOsmol/kg $\mathrm{H}_{2} \mathrm{O}$ ) & $288(285 ; 291)$ & $297(292 ; 307)$ & $294(290 ; 301)$ & $306(303 ; 312)$ & $<0.001$ \\
\hline Diuretics & $0(0 \%)$ & $1(6.7 \%)$ & $0(0 \%)$ & $0(0 \%)$ & 0.54 \\
\hline Lithium & $0(0 \%)$ & $0(0 \%)$ & $1(5.3 \%)$ & $5(71.4 \%)$ & $<0.001$ \\
\hline Antiepileptics & $0(0 \%)$ & $1(6.7 \%)$ & $2(10.5 \%)$ & $2(28.6 \%)$ & 0.29 \\
\hline Corticosteroids & $0(0 \%)$ & $6(40 \%)$ & $2(10.5 \%)$ & $0(0 \%)$ & 0.06 \\
\hline Desmopressin & $0(0 \%)$ & $9(60 \%)$ & $0(0 \%)$ & $0(0 \%)$ & $<0.001$ \\
\hline Birth control pill (\% of females) & $0(0 \%)$ & $2(22 \%)$ & $3(23 \%)$ & $0(0 \%)$ & $<0.001$ \\
\hline
\end{tabular}

Table 2: Plasma apelin and copeptin levels

\begin{tabular}{lccccc} 
& $\begin{array}{c}\text { Healthy } \\
\text { volunteers } \\
(\mathbf{n = 1 1 3 )}\end{array}$ & $\begin{array}{c}\text { Complete } \\
\text { central DI } \\
(\mathbf{n = 1 5 )}\end{array}$ & $\begin{array}{c}\text { Primary } \\
\text { polydipsia } \\
(\mathbf{n = 1 9 )}\end{array}$ & $\begin{array}{c}\text { Complete } \\
\text { nephrogenic DI } \\
(\mathbf{n = 7})\end{array}$ & p-value \\
\hline Plasma copeptin (pmol//) & $4.1(2.6 ; 6.9)$ & $1.9(1.9 ; 2.5)$ & $3.9(2.2 ; 4.7)$ & $56.7(39.7 ; 70)$ & $<0.001$ \\
Plasma apelin (pmol/I) & $254(225 ; 311)$ & $209(174 ; 241)$ & $190(172 ; 215)$ & $413(332 ; 504)$ & $<0.001$ \\
Plasma apelin/copeptin (pmol/pmol) & $57(37 ; 102)$ & $89(73 ; 135)$ & $53(38 ; 92)$ & $7(6 ; 10)$ & $<0.001$
\end{tabular}

Figure 1: Plasma apelin concentrations

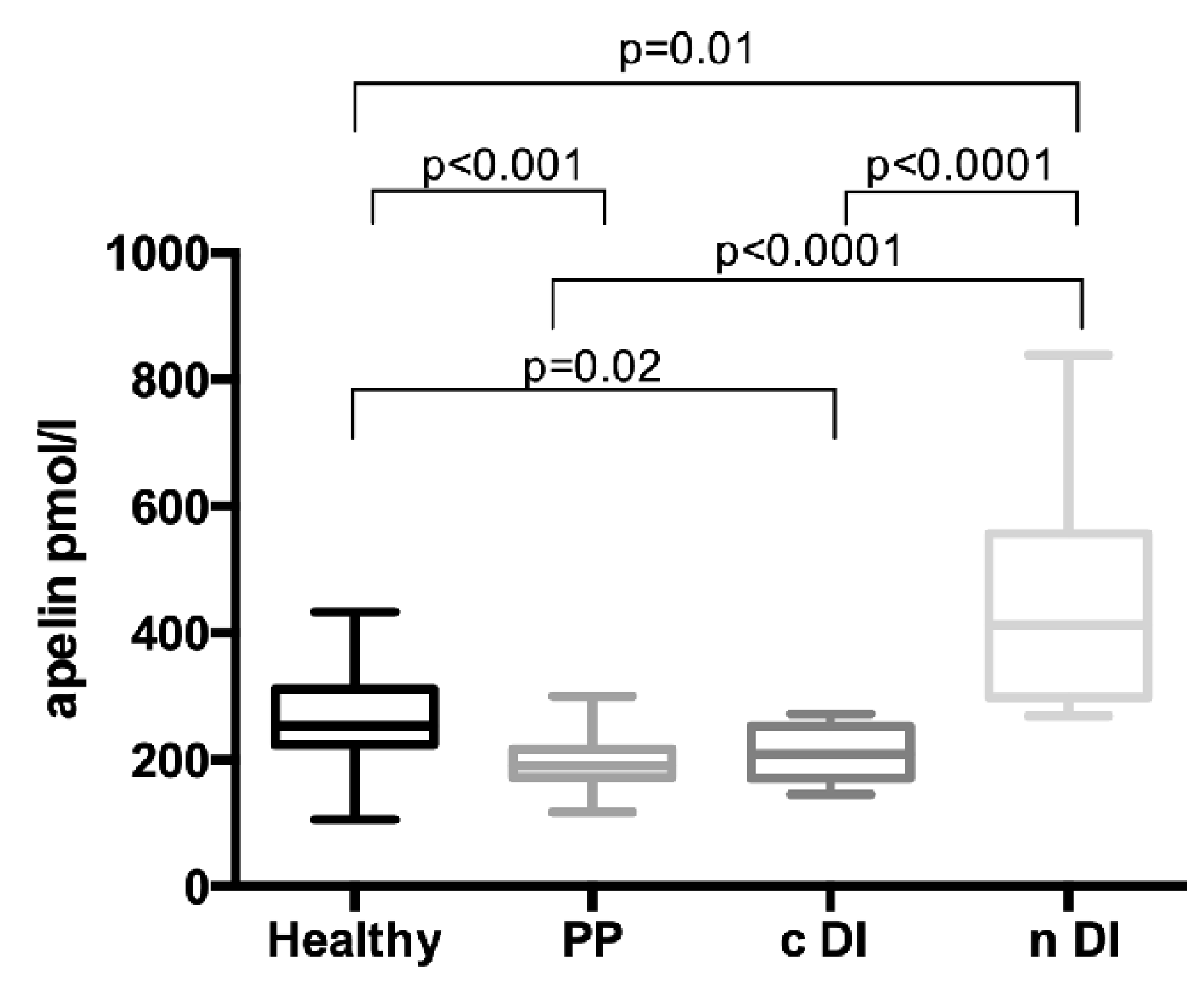

Figure 2: Plasma apelin to copeptin ratios

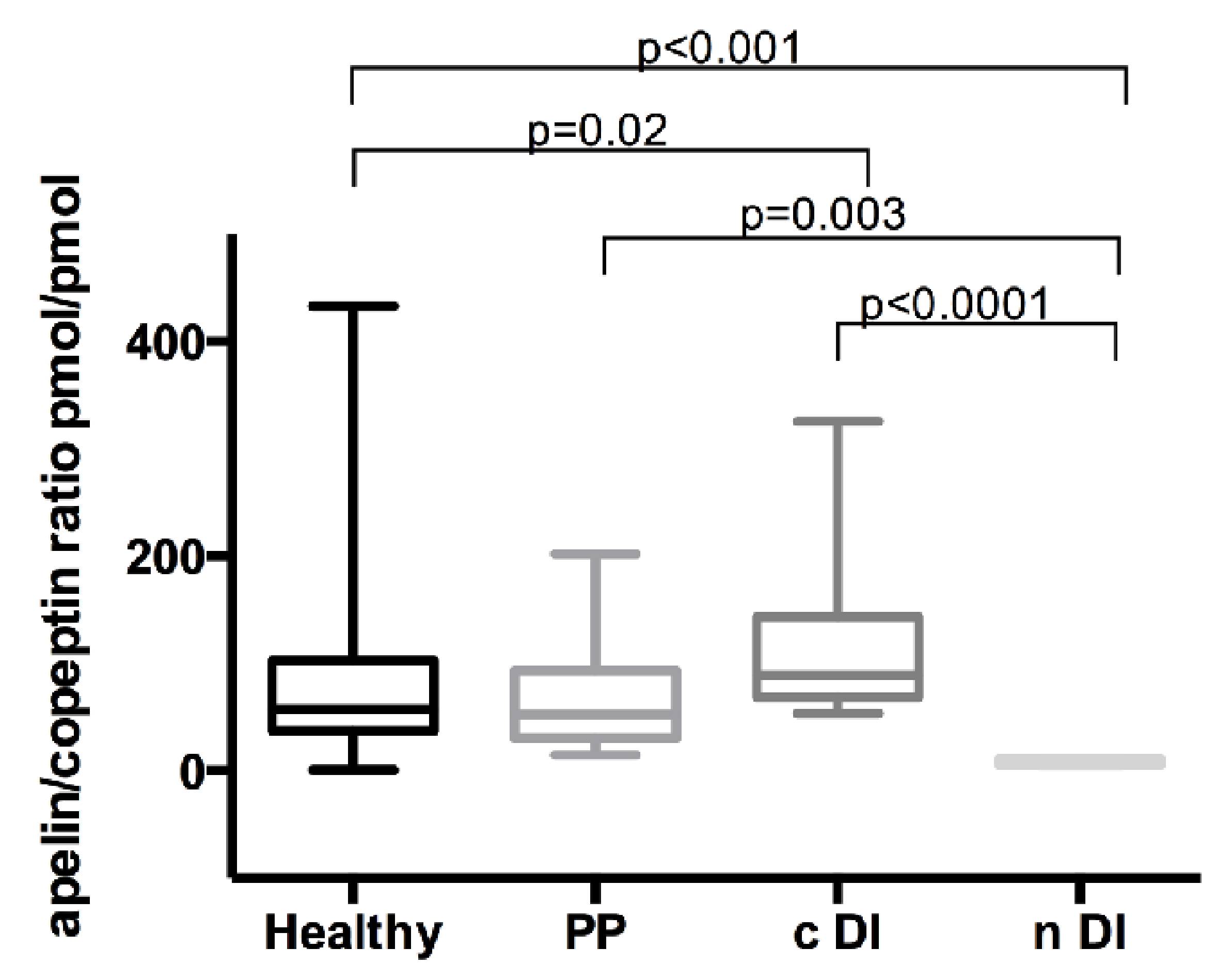

\section{Summary \& Conclusion}

In primary polydipsia, normal plasma apelin to copeptin ratio attests a normal water homeostasis.

In contrast, in patients with central or nephrogenic DI the increased or decreased apelin to copeptin ratio, respectively,

reflects a disturbed osmotic and body fluid homeostasis.

References

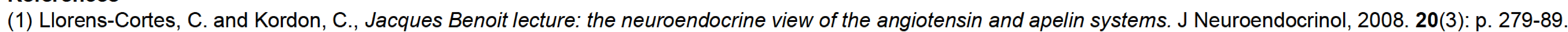

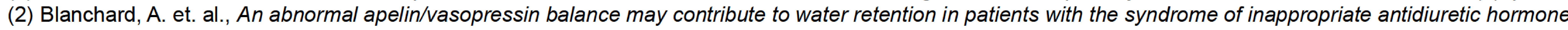

(SIADH) and heart failure. JCEM. 2013;98:2084-2089. 